\title{
GALAXY-WIDE SHOCKS IN LATE-MERGER STAGE LUMINOUS INFRARED GALAXIES
}

\author{
J. A. RiCH ${ }^{1}$, L. J. KewLeY ${ }^{1}$, AND M. A. Dopita ${ }^{1,2}$ \\ ${ }^{1}$ Institute for Astronomy, University of Hawaii, 2680 Woodlawn Drive, Honolulu, HI 96822, USA; jrich@ifa.hawaii.edu \\ ${ }^{2}$ Research School of Astronomy and Astrophysics, Australian National University, Cotter Rd., Weston ACT 2611, Australia \\ Received 2011 January 30; accepted 2011 April 5; published 2011 May 27
}

\begin{abstract}
We present an integral field spectroscopic study of two nearby luminous infrared galaxies (LIRGs), IC 1623 and NGC 3256, which exhibit evidence of widespread shock excitation induced by ongoing merger activity. We show the importance of carefully separating excitation due to shocks versus excitation by $\mathrm{H}$ II regions and the usefulness of integral field unit data in interpreting the complex processes in LIRGs. Our analysis focuses primarily on the emission line gas, which is extensive in both systems and is a result of the abundant ongoing star formation as well as widespread LINER-like excitation from shocks. We use emission line ratio maps, line kinematics, line-ratio diagnostics, and new models as methods for distinguishing and analyzing shocked gas in these systems. We discuss how our results inform the merger sequence associated with local U/LIRGs and the impact that widespread shock excitation has on the interpretation of emission line spectra and derived quantities of both local and high-redshift galaxies.
\end{abstract}

Key words: galaxies: evolution - galaxies: individual (NGC 3256, IC 1623) - galaxies: ISM - shock waves

Online-only material: color figures

\section{INTRODUCTION}

Understanding the physical processes involved in the formation and evolution of galaxies remains a key problem in modern astrophysics. As observers strive to interpret data on the first generations of galaxies and theoreticians attempt to recreate the assembly and evolution of galaxies, studies of the nearby universe are needed to constrain key physical processes and global parameters that inform both observations and models of galaxies in the distant universe. In this regard, luminous infrared galaxies (LIRGs) and ultraluminous infrared galaxies (ULIRGs) provide important observational constraints to models of distant galaxies. Although rare locally, IR-luminous galaxies and the merger activity associated with this class of systems become increasingly important at high redshift, contributing with U/LIRGs, the overwhelmingly dominant component of the IR luminosity function by $z=1$ (Elbaz et al. 2002; Le Floc'h et al. 2005). Not only does the improved spatial resolution allow us to study the relative importance of physical processes in the various components of the U/LIRG system, but also we can study the physics over the full array of merger states. Many recent studies across multiple wavelengths are beginning to focus on resolving the detailed ionization structure, kinematics, and power sources of local U/LIRGs in order to further our understanding of galaxy evolution both locally and at high redshift.

The local population of LIRGs (which range from $10^{11} L_{\odot}<$ $L_{\mathrm{IR}}<10^{12} L_{\odot}$ ) have been found to represent a variety of objects from particularly dusty isolated star-forming galaxies to progressed mergers or late-stage mergers. Most frequently, however, U/LIRGs are often associated with merger activity which drives the intense circumnuclear star formation that fuels the powerful IR luminosity (Veilleux et al. 1995; Scoville et al. 2000; Arribas et al. 2004; Alonso-Herrero et al. 2006). The gas in the outskirts of each system is torqued into the center which drives the ongoing star formation as the merger continues (Barnes \& Hernquist 1996). As the galaxies move along the Toomre \& Toomre (1972) merger sequence, the IR luminosity is generally found to increase along with galactic wind activity (Rupke et al. 2005). At the terminal phase of the merger sequence, galaxies have in general become ULIRGs and appear as a coalesced or nearly coalesced system. Finally, these become dominated by a powerful active galactic nucleus (AGN) as their star formation becomes quenched as the available gas reservoir becomes depleted (Genzel et al. 1998; Yuan et al. 2010).

As this merger sequence progresses, tidally induced gas motions and outflows from galactic winds become increasingly common. These forces can have an influence on the emission line gas through shocks that are induced by the large-scale gas flows (Armus et al. 1989; Heckman et al. 1990; Lehnert \& Heckman 1996; Colina et al. 2005; Zakamska 2010). This shock excitation may contaminate line ratios used to determine metallicity, star formation rate (SFR), and power source in a galaxy. Depending on the geometry and kinematics of the gas and dust, the ionization of the observed emission line gas can be dominated by shock excitation induced by either tidal flows (Colina et al. 2005; Farage et al. 2010; Monreal-Ibero et al. 2010) or galactic winds (Veilleux \& Rupke 2002; Veilleux et al. 2003; Lípari et al. 2004a; Sharp \& Bland-Hawthorn 2010; Rich et al. 2010; Moiseev et al. 2010). Early work using narrow band or tunable filters and Fabry-Perot instruments proved useful for investigating these processes over large swaths of a galaxy (Shopbell \& Bland-Hawthorn 1998; Veilleux \& Rupke 2002; Calzetti et al. 2004). However, a detailed characterization of the shock excitation and its effects on emission line spectra is more complex, necessitating the use of integral field spectroscopy (IFS).

The advent of integral field units (IFUs) has led to a rapid multiplication of observational evidence for extended shock excitation in nearby galaxies. Sharp \& Bland-Hawthorn (2010) conducted a study of several galactic winds and found widespread shock excitation in the extended emission associated with the outflow. Monreal-Ibero et al. (2006) identified extended low-ionization narrow emission-line region (LINER)like emission resulting from shocks in a small IFU sample of ULIRGs and followed up with a more thorough IFU study of tidally induced shock excitation in nearby LIRGs (Monreal-Ibero et al. 2010). Farage et al. (2010) discovered 


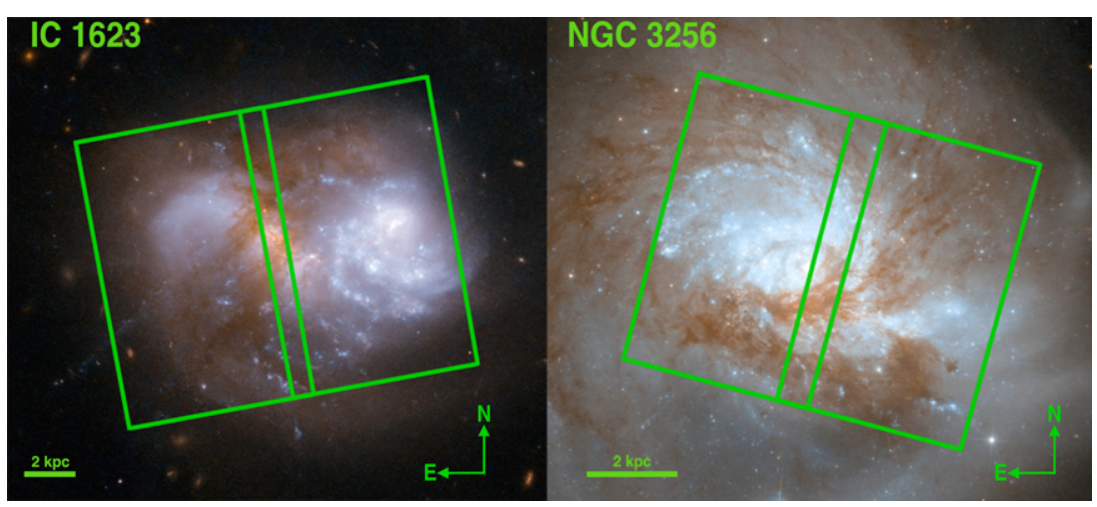

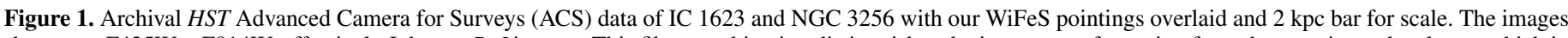

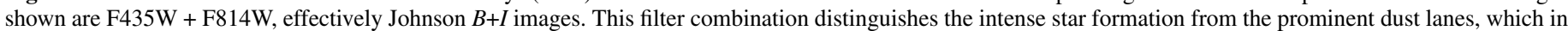
both galaxies heavily obscure the secondary nucleus of the merging pair.

(A color version of this figure is available in the online journal.)

extended shocks caused by gas accreting onto a giant brightest cluster galaxy. Finally, Rich et al. (2010) found extended shock excitation caused by a galactic wind in the M82-like galaxy NGC 839. In both Farage et al. (2010) and Rich et al. (2010), new slow shock models were employed to analyze the shocked gas. In all of the above cases shock excitation exhibits characteristics of extended LINER-like emission.

In this paper, we present a detailed analysis of the widespread shock excitation in two galaxies drawn from a larger IFU sample of nearby U/LIRGs: the Wide Field Spectrograph (WiFeS) Great Observatory All-Sky LIRG Survey (GOALS) sample. We discuss these two systems, IC 1623 and NGC 3256, in Section 2, and our observations, data reduction, and line fitting in Section 3. We present the properties of the emission line gas for these two systems in Section 4, including line ratio maps in Section 4.1 and emission line diagnostic diagrams in Section 4.2. We discuss the distribution of velocity dispersions for each system in Section 5. We combine the observed quantities and use them to separate the shocked gas from the $\mathrm{H}$ II region gas and provide an analysis of our results in Section 6. In Section 7, we discuss the implications of our results for the interpretation of emission line spectra and investigate the effect that extended shock excitation can have on quantities derived from single aperture spectra of nearby and high-redshift galaxies. Finally, we give our conclusions in Section 8.

Throughout this paper we adopt the cosmological parameters $H_{0}=70 \mathrm{~km} \mathrm{~s}^{-1} \mathrm{Mpc}^{-1}, \Omega_{\mathrm{V}}=0.72$, and $\Omega_{\mathrm{M}}=0.28$, based on the five-year Wilkinson Microwave Anisotropy Probe results (Hinshaw et al. 2009) and consistent with the Armus et al. (2009) summary of the GOALS sample. Systemic heliocentric velocities adjusted using the 3-attractor flow model of Mould et al. (2000) are taken from Armus et al. (2009).

\section{SAMPLE}

The systems analyzed in this paper are drawn from a larger integral field spectroscopic survey of the GOALS sample. GOALS is a multi-wavelength survey of the brightest $60 \mu \mathrm{m}$ extragalactic sources in the local universe $\left(\log \left(L_{\mathrm{IR}} / L_{\odot}\right)>11.0\right)$, with redshifts $z<0.088$ and is a complete subset of the IRAS Revised Bright Galaxy Sample (Sanders et al. 2003). Objects in GOALS cover the full range of nuclear spectral types and interaction stages and provide excellent nearby laboratories for the study of galaxy evolution.
Table 1

Information for the Two Systems Observed for This Paper

\begin{tabular}{lccccc}
\hline \hline Galaxy & $z$ & R.A. & Decl. & $B$ Mag. & $\log \left(L_{\mathrm{IR}} / L_{\odot}\right)$ \\
\hline IC 1623 & 0.0201 & $01: 07: 47$ & $-17: 30: 27$ & 15.04 & 11.71 \\
NGC 3256 & 0.00935 & $10: 27: 51$ & $-43: 54: 14$ & 11.83 & 11.64 \\
\hline
\end{tabular}

Notes. Names, systemic redshift, and IR luminosities are taken from the Armus et al. (2009) summary of the GOALS sample. R.A. and decl. given are the center of our mosaiced WiFeS pointings. $B$ magnitudes are from the surface photometry catalog of the ESO-Uppsala Galaxies.

We chose IC 1623 and NGC 3256 for a more detailed analysis since they both show evidence of widespread shock excitation. Table 1 shows the global properties of the two systems, both of which are ongoing mergers of two massive spiral galaxies. Both systems are fairly luminous LIRGs and neither shows evidence of an embedded AGN. Archival Hubble Space Telescope (HST) data of the systems are shown in Figure 1. Both systems show a complex optical morphology and contain one galaxy nucleus that is heavily extinguished by dust lanes. Both galaxies are classified as close mergers (projected separation between the merging nuclei of less than $10 \mathrm{kpc}$ ) when using the scheme of Veilleux \& Rupke (2002), which utilizes classifications based on numerical simulations of mergers (Barnes \& Hernquist 1992, 1996).

IC 1623. IC 1623 (also commonly referred to as VV 114 and with the IRAS designation IRASF 01053-1746) consists of two galaxies: IC $1623 \mathrm{~A}$ in the west and the more heavily obscured IC 1623 B in the east. The dust lane seen in Figure 1 covers the eastern nucleus, which is actually brighter in the near-IR than the western disk (Knop et al. 1994; Doyon et al. 1995; Scoville et al. 2000). The galaxy nuclei are separated by approximately $5 \mathrm{kpc}$, firmly classifying the pair as a close merger in the Veilleux \& Rupke (2002) classification scheme. Interestingly, the heavily obscured eastern galaxy has two very bright sources of similar magnitude in the near-IR, raising the possibility that IC $1623 \mathrm{~B}$ itself is a merger. However, it may be that there is simply a second region of heavily obscured star formation unassociated with the true nucleus. Because of the extensive dust lane, the majority of the optical emission in our observations is dominated by the star formation in the western galaxy. Neither of the galaxies exhibits evidence of an AGN, obscured or otherwise (Doyon et al. 1995; Veilleux et al. 1995). Extended tidal features can be seen in optical and 
near-IR imaging, though the low surface brightness of these features makes observations of them prohibitively time-consuming. The galaxy pair is at a redshift of $z \sim 0.0201$, corresponding to a $1^{\prime \prime}$ projected distance of $\sim 385 \mathrm{pc}$.

$N G C$ 3256. NGC 3256 (IRAS designation IRASF102574339 ) is a late-stage merger with nuclei separated by approximately $1 \mathrm{kpc}$, though it has not yet formed a single nucleus and therefore cannot be classified as a post-merger. Sakamoto et al. (2006) note that the long tidal tails seen in optical and $\mathrm{H}$ I images (de Vaucouleurs \& de Vaucouleurs 1961; English et al. 2003) suggest "a prograde-prograde merger of two gas-rich spiral galaxies of similar size" (Toomre \& Toomre 1972; White 1979). The bright face-on galaxy seen in Figure 1 has a separated, heavily obscured secondary nucleus seen in X-ray, IR, and radio lying $\sim 5^{\prime \prime}$ south of the bright optical nucleus (Boeker et al. 1997; Neff et al. 2003; Sakamoto et al. 2006). Neff et al. (2003) posited that the two nuclei may be low-luminosity AGNs based on the radio to X-ray ratios, though they also note that a large number of supernova remnants could also account for the observed ratio. However, subsequent observations by Lípari et al. (2004b) of galactic bubbles associated with previous supernovae in the core of NGC 3256 support the latter case and rule out any significant contribution from even a low-luminosity AGN. This supernova activity in the core of NGC 3256 is also driving a galactic wind measured in neutral absorption and ionized emission previously associated with shock heating (Armus et al. 1989; Heckman et al. 1990, 2000; Lípari et al. 2000). NGC 3256 lies closer than IC 1623 at $z \sim 0.0094$, which means that our data probe a finer spatial scale of $1^{\prime \prime} \sim 185 \mathrm{pc}$.

\section{OBSERVATIONS AND DATA REDUCTION}

Our data were taken with the WiFeS instrument at the Mount Stromlo and Siding Spring Observatory $2.3 \mathrm{~m}$ telescope. WiFeS is a dual beam, image-slicing integral field spectrograph commissioned in 2009 May and described in detail by Dopita et al. (2007) and Dopita et al. (2010). Our data consist of separate blue and red spectra with wavelength coverage of $\sim 3500-5800 \AA$ and $\sim 5500-7000 \AA$, respectively, and a resolution of $R=3000$ (blue) and $R=7000$ (red), corresponding to a velocity resolution of $100 \mathrm{~km} \mathrm{~s}^{-1}$ at $\mathrm{H} \beta$ and $40 \mathrm{~km} \mathrm{~s}^{-1}$ at $\mathrm{H} \alpha$. Observations of IC 1623 were carried out on 2009 August 20. Two separate pointings were observed for 40 minutes in total for each pointing. NGC 3256 was observed on 2010 March 15 with two individual pointings for 60 minutes each. Each galaxy was observed using two pointings as shown in Figure 1, with a large enough overlap region to facilitate mosaicing. The effective field of view of the data in both cases is thus approximately $45^{\prime \prime} \times 38^{\prime \prime}$, providing well over 1000 spectra in each set of data cubes.

The data were reduced and flux calibrated using the WiFeS pipeline, briefly described in Dopita et al. (2010), which uses IRAF routines adapted primarily from the Gemini NIFS data reduction package. A single WiFeS observation consists of 25 slit spectra (and an additional 25 contemporaneous sky spectra if the data were taken in nod and shuffle mode). Each individual observation was reduced into a data cube using the process described below.

Bias subtraction is slightly complicated due to temporal changes in the quad-readout bias levels and a slope in the bias level on each WiFeS chip. Bias frames are taken immediately before or after each observation or set of observations and a two-dimensional fit of the surface of this bias frame is subtracted from the temporally nearest object data in order to avoid adding additional noise to the data. Any resulting residual in the bias level is accounted for with a fit to unexposed regions of the detector.

Quartz lamp flats are used to remove pixel-to-pixel variations from the response curve of the chip and twilight sky flats are used to correct for illumination variation along each of the slitlets. Spatial calibration is carried out by placing a thin wire in the filter wheel and illuminating the slitlet array with a continuum lamp. This procedure defines the center of each slitlet. The individual spectra have no spatial distortion because the camera corrects the small amount of distortion introduced by the spectrograph. Thus, only low-order mapping of the slitlets is required.

$\mathrm{CuAr}$ and NeAr arc lamps are used to wavelength calibrate the blue and red spectra, respectively. Arc lamp data were taken throughout each night of observing to account for any change in the wavelength solution. Each of the 25 slitlets is then rectified by the pipeline into a full data cube (one for each arm) and sampled to a common wavelength scale for each target. The spectral resolution achieved within the red data cubes is $46 \pm 6 \mathrm{~km} \mathrm{~s}^{-1}$. We use this value to remove instrumental broadening from the derived velocity dispersions.

Telluric absorption features were removed from the resulting red data cubes using observations of B-stars or featureless white dwarfs taken at similar air mass. The effects of differential atmospheric refraction are calculated and corrected by the pipeline for the blue data cubes.

Flux calibration of each individual data cube was carried out using flux standards observed throughout each night. The white dwarf EG 131 was used to flux calibrate IC 1623 and the white dwarf L745-46a was used to flux calibrate NGC 3256 (Bessell 1999).

Finally, the individual reduced, flux-calibrated data cubes are median combined and sampled to a common spatial grid using overlapping features found in each pointing. Observations of NGC 3256 were binned on-chip by 2 pixels in the spatial direction in order to increase the signal-to-noise ratio $(\mathrm{S} / \mathrm{N})$ and produce square spatial elements $1^{\prime \prime} \times 1^{\prime \prime}$. Observations of IC 1623 were binned after reduction for the same reasons. This means that for both systems the scale of the data cubes and of the resulting maps presented in this paper are $1^{\prime \prime}$ spaxel $^{-1}$.

Combined data cubes were aligned astrometrically by comparing a pseudo " $r$-band" image generated using the red spectrum from each spaxel with HST images from the archive.

\subsection{Spectral Fitting}

We analyzed every spectrum using an automated fitting routine written in IDL, UHSPECFIT, which is based on the code created by Jabran Zahid et al. (2011) and is also employed by and described in Rupke et al. (2010b) and Rich et al. (2010). Our routine fits and subtracts a stellar continuum from each spectrum using population synthesis models from González Delgado et al. (2005) and an IDL routine which fits a linear combination of stellar templates to a galaxy spectrum using the method of Moustakas \& Kennicutt (2006).

After the continuum is removed, lines in the resulting emission spectra are fit using a one- or two-component Gaussian, depending on the goodness of fit determined by the routine. Resulting fits were inspected to ensure the fitting code did not fail. All emission lines are fit simultaneously using the same Gaussian component or components. Both continuum and emission lines were fit using the MPFIT package, which performs a least-squares analysis using the Levenberg-Marquardt algorithm (Markwardt 2009). Example fits are provided in Figure 2. 


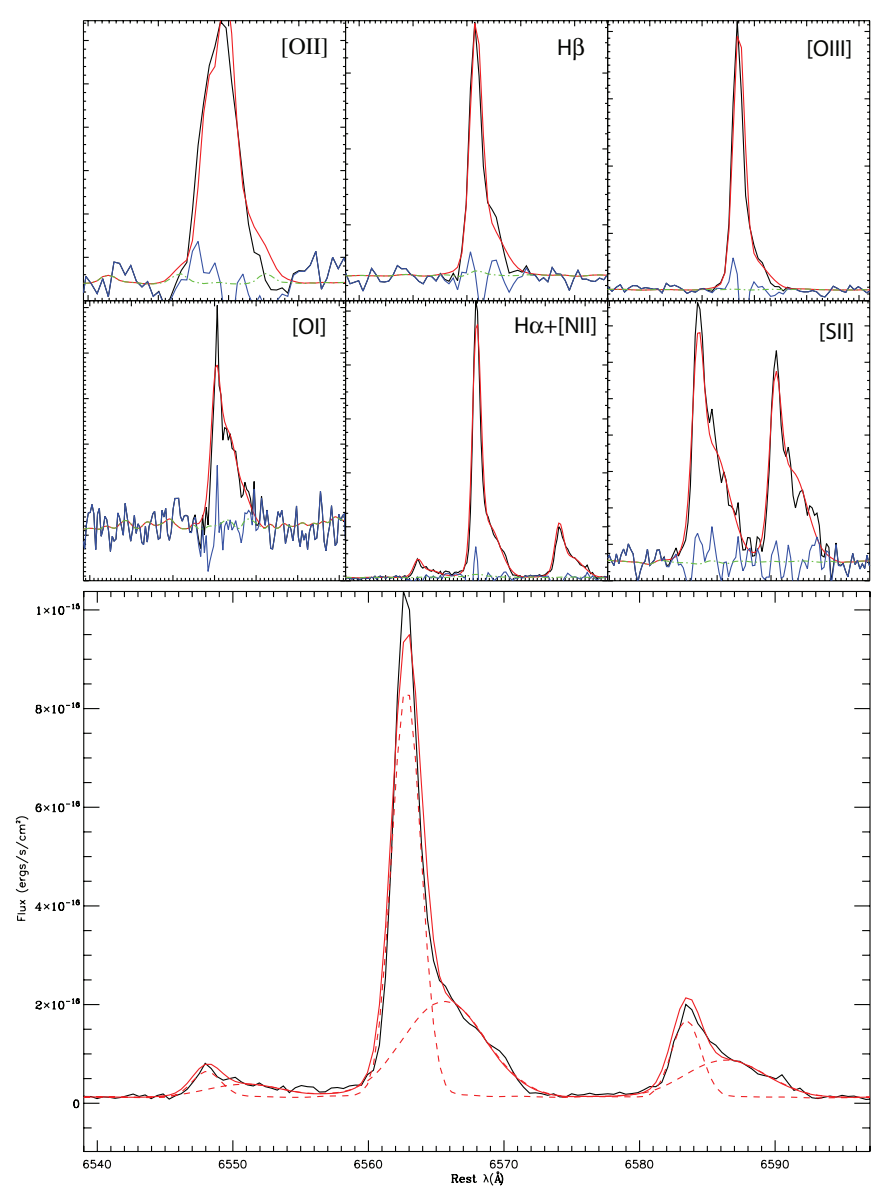

Figure 2. Example of emission line fitting routine results for a single spectrum from IC 1623. The upper panels show individual emission lines of interest with data in black, total fit in red, continuum in green, and residuals in blue. The bottom panel shows a zoomed-in region around $\mathrm{N}$ II and $\mathrm{H} \alpha$ with each Gaussian component plotted as a dotted line and the total fit plotted as a solid line. Wavelengths are plotted in the rest frame with respect to the narrow component.

(A color version of this figure is available in the online journal.)

The errors in the parameters used in fitting the emission lines are calculated by the fitting code. This includes the Gaussian widths (velocity dispersions) and ratio of dispersions between the blue and red spectra, individual Gaussian peaks, redshift and any slight deviation between the blue and red wavelength calibrations. Emission line fluxes used in derived quantities are subject to a cut of minimum $\mathrm{S} / \mathrm{N}$ of 5 , which is calculated by comparing the peak of the Gaussian to the noise in the continuum. The lower resolution of the blue spectra contributes to the uncertainty of the Gaussian parameters-for instance in some cases one or two emission line components for a blue emission line may provide a similar goodness of fit. By minimizing the $\chi^{2}$ for all of the lines simultaneously we increase slightly the overall uncertainty in the velocity dispersion while maintaining the ability to analyze multiple components fits.

In many cases it is crucial that more than one Gaussian is used in the emission line fit. Our analysis relies on a careful decomposition of multiple velocity profiles, which are common in the kinematically complex family of U/LIRGs. An example can be seen in Figure 2, where two distinct components have been fit. In the case shown, there is an obvious redshifted component with a broader profile than the primary component. The line ratios between the two components are noticeably different as well.

\section{EMISSION LINE GAS PROPERTIES}

Emission line ratios have proven a useful tool for probing the sources of ionizing radiation in galaxies. The primary factors influencing emission line strengths are the shape of the extreme ultraviolet spectrum and the abundance of the emission line gas (Kewley et al. 2001; Dopita et al. 2000). Thus, with IFU data a line ratio map and diagnostic diagram can be created as a useful tool in interpreting the power sources not just in the nucleus of a galaxy but also across the galaxy as a whole.

\subsection{Line Ratio Maps}

In Figure 3, we examine maps of the ratios of the total flux of [N II] $6583 \AA$, [S II] 6717,6731 are sensitive to metallicity as well as the ionization parameter as a first step toward understanding the processes at work in IC 1623 and NGC 3256. Line ratio values corresponding to each spaxel value are also plotted on standard diagnostic diagrams in Figure 4.

IC 1623. Most of the optical emission line flux in IC 1623 is dominated by the eastern galaxy (IC $1623 \mathrm{~A}$ ), which is less extinguished by dust. The emission line ratios in this portion of the merging system are lower than in the rest of the system, tracing the star formation evidenced by the less extinguished $\mathrm{H}$ II regions and clusters seen in the archival $H S T$ images. There is no evidence of a metallicity gradient in the $[\mathrm{N}$ II $] / \mathrm{H} \alpha$ ratio map, which is in agreement with recent work on the flattening of metallicity gradients in merging systems by Rupke et al. (2010a, 2010b) and Kewley et al. (2010).

Moving outward from the main regions of unobscured star formation in IC 1623 A we see an overall rise in all line ratios. Toward the northern and southern parts of IC1623 A, the line ratios increase to levels inconsistent with excitation by $\mathrm{H}$ II regions alone until the $\mathrm{S} / \mathrm{N}$ in emission is too low. Especially striking are the high line ratios correlated with the dust lane east of IC 1623 A which obscures the better part of IC $1623 \mathrm{~B}$. The emission line ratios show consistently high values across this dust lane, especially in $[\mathrm{O} \mathrm{I}] / \mathrm{H} \alpha$, which is a particularly good tracer of shock excitation (Farage et al. 2010; Rich et al. 2010). The total [O I] flux in this region approaches $1 / 4$ to $1 / 3$ the total $\mathrm{H} \alpha$ flux, while the total $\mathrm{N}$ II flux is as much as $1 / 2$ to $2 / 3$ the total $\mathrm{H} \alpha$ flux.

NGC 3256. The line ratio maps of NGC 3256 show similar behavior compared to IC 1623. Again the areas of unobscured star formation correspond to lower line ratios, tracing the ongoing star formation as the dominant source of ionizing photons. A key difference is that NGC 3256 shows a region of increasing line ratios in a small central part of the region of low line ratios. This increase corresponds to the previously observed galactic wind in NGC 3256 (Heckman et al. 1990) and our spectra in this region exhibit strong, broad blueshifted $\mathrm{NaD}$ profiles (Figure 5). The increasing emission line ratios in this case are due to the additional presence of shock excitation from the galactic wind.

Outside of both the central region influenced by the galactic wind and the primarily star formation dominated portions of the galaxy the line ratios again tend to increase, with $[\mathrm{N}$ II $] / \mathrm{H} \alpha$ reaching nearly unity and correspondingly high $[\mathrm{S}$ II $] / \mathrm{H} \alpha$ and $[\mathrm{O} \mathrm{I}] / \mathrm{H} \alpha$ in the outer spatial portions of our data cubes. These regions of strong excitation correlate strongly with dust lanes evident in the HST archival data and are quite distant from the core of the galaxy. 

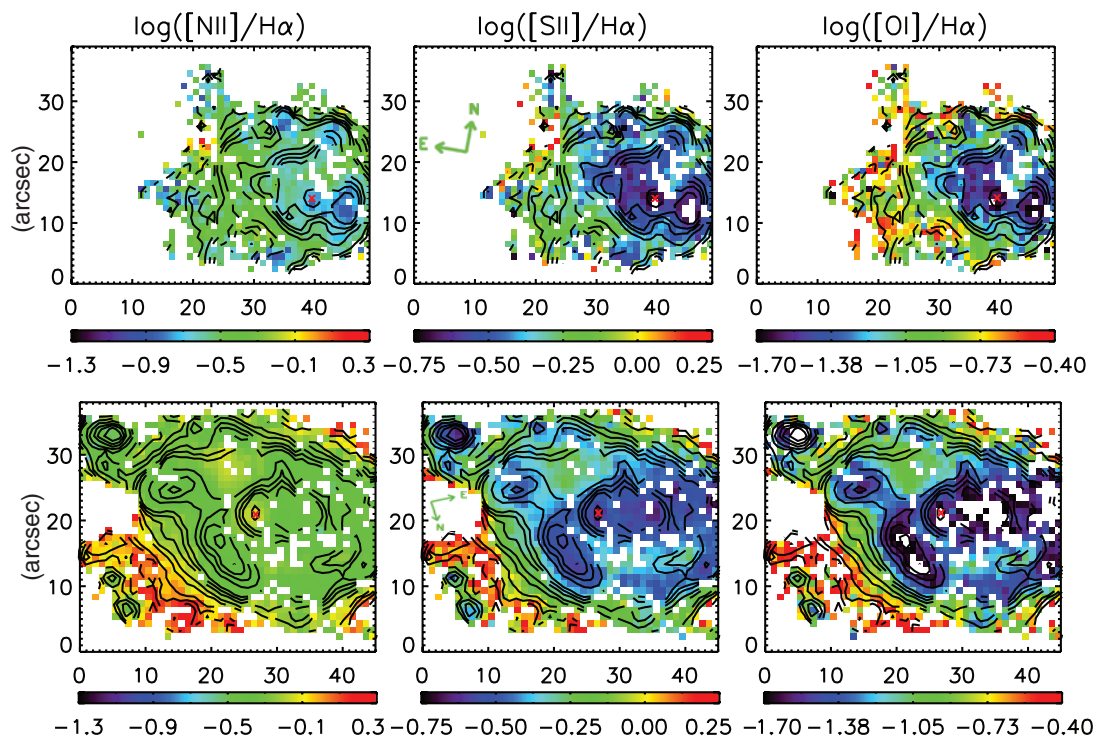

Figure 3. From left to right, emission line ratio maps of $[\mathrm{N} \mathrm{II}] / \mathrm{H} \alpha$, [S II] $/ \mathrm{H} \alpha$, and $[\mathrm{OI}] / \mathrm{H} \alpha$ for IC 1623 (top) and NGC 3256 (bottom). $\mathrm{H} \alpha$ contours have been overlaid, and the peak in $\mathrm{H} \alpha$ flux has been marked with a small red " $\times$." These maps are a sum of the total emission line flux in both profiles and trace the dominant ionization mechanism in different portions of the galaxy. Generally speaking, the stronger the line ratio the further the departure from an ionizing spectrum purely due to star formation. The regions most dominated by ongoing star formation appear bluer in the above maps. Maps are oriented with respect to the original observations (cf. Figure 9).

(A color version of this figure is available in the online journal.)

Line Ratios for single and double components

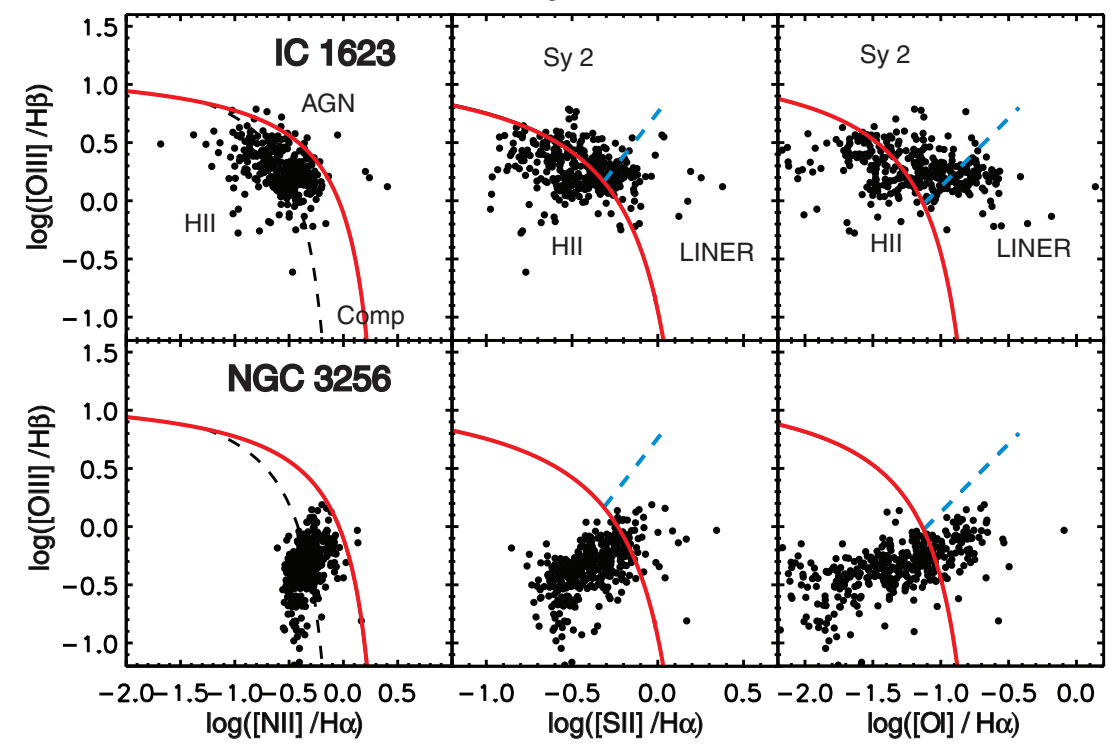

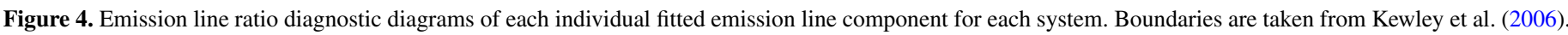

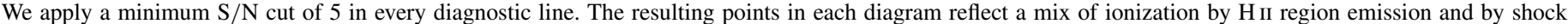

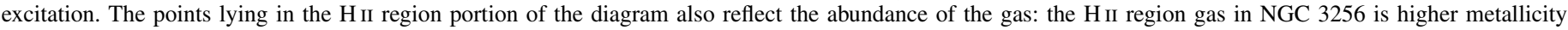
resulting in a different overall shape to the distribution of points in the diagnostic diagram.

(A color version of this figure is available in the online journal.)

\subsection{Diagnostic Diagrams}

In order to better understand the power sources at play, we turn to line ratio diagnostic diagrams. Diagnostics using $[\mathrm{N}$ II $] / \mathrm{H} \alpha,[\mathrm{S} \mathrm{II}] / \mathrm{H} \alpha$ or $[\mathrm{O} \mathrm{I}] / \mathrm{H} \alpha$ ratios against the $\left[\mathrm{O}{ }_{\mathrm{III}}\right] / \mathrm{H} \beta$ ratio were first employed by Baldwin et al. (1981) and Veilleux \& Osterbrock (1987) to distinguish the source of ionizing photons in global or nuclear galaxy spectra. This diagnostic scheme has been successfully applied to integral field spectra (as well as narrowband and tunable filter data) to investigate emission line gas over entire galaxies or regions of a galaxy to probe the distribution of ionizing sources (e.g., Shopbell \& Bland-Hawthorn 1998; Sharp \& Bland-Hawthorn 2010; Rich et al. 2010). To separate the ionizing sources with IFU data, individual spectra from each spaxel are plotted on the traditional diagnostic diagrams. In this way the ensemble of points provides information about the various processes at work in different portions of the galaxy rather than a single global measure.

We plot diagnostic diagrams in Figure 4 and include all emission line components from each spaxel with $\mathrm{S} / \mathrm{N}>5$ in all six diagnostic lines $(\mathrm{H} \beta, \mathrm{H} \alpha$, [O III] $5007 \AA$, [O I] $6300 \AA$, [N II] $6583 \AA$, [S II] 6717,6731 $⿱$ A). In all diagrams, the lower 

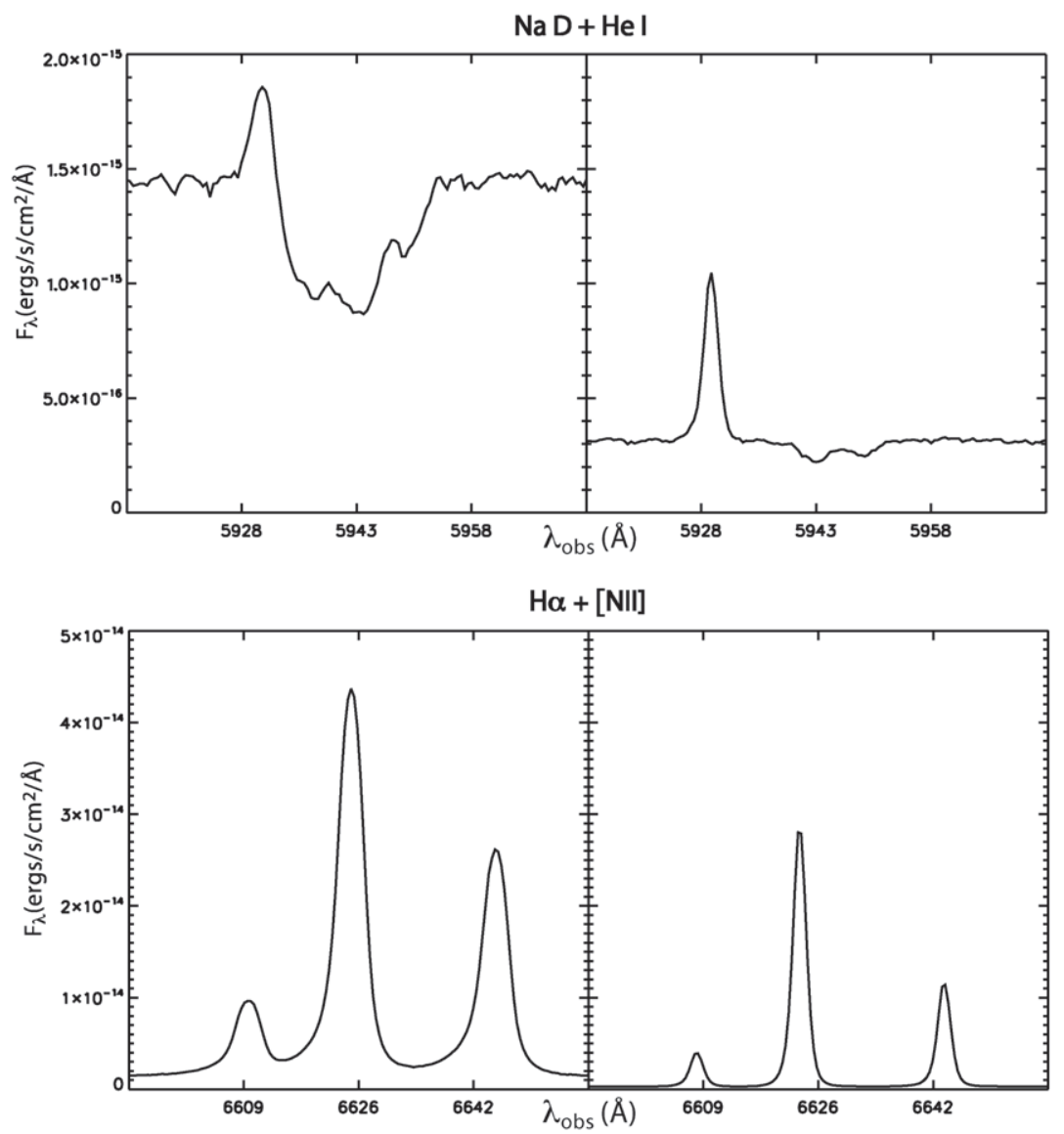

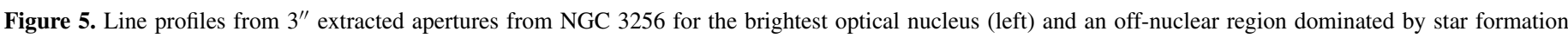

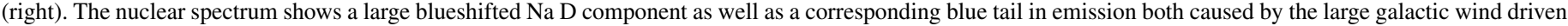
by the nuclear starburst.

left-hand portion of the plot traces photoionization by $\mathrm{H}$ II regions. The solid curved line traces the upper theoretical limit to pure $\mathrm{H}_{\mathrm{II}}$ region contribution measured by Kewley et al. (2001) and the dotted line in the $\mathrm{N}$ II diagnostic provides an empirical upper limit to the H II region sequence of Sloan Digital Sky Survey (SDSS) galaxies measured by Kauffmann et al. (2003). The region lying between these two lines represents objects with a composite spectrum mixing $\mathrm{H}$ II region emission with a stronger ionizing source. Contribution from a Seyfert AGN pushes points into the upper region of the diagrams while more LINER-like emission lies to the right-hand side of the diagrams, as shown by the dividing line in the $[\mathrm{S} \mathrm{II}]$ and [O I] diagnostics, as established in Kewley et al. (2006). New lower velocity shock models of order $100-200 \mathrm{~km} \mathrm{~s}^{-1}$ trace line ratios from the upper edge of HII-like emission into the LINER region of the diagnostic diagrams (Farage et al. 2010; Rich et al. 2010).

\section{VELOCITY DISPERSIONS}

Where more than one component is fit to emission lines ordering the components over a whole IFU data cube is nontrivial. The difference in velocity dispersion and flux where two components are fit varies depending on the conditions in the region of the galaxy observed and is dependent on several factors including the relative flux, source of ionization, and extinction within each component. In some cases two components of comparable flux and velocity dispersion are observed, while in other regions two components of significantly different flux, flux ratios, and velocity dispersion are seen (cf. Figure 2). In this section, we focus on the velocity dispersion of individual emission line components as the most useful way of probing the multiple components observed.

If shock excitation is important in these regions showing enhanced $[\mathrm{O} \mathrm{I}] / \mathrm{H} \alpha,[\mathrm{N} \mathrm{II}] / \mathrm{H} \alpha$, and $\left[\mathrm{S}_{\mathrm{II}}\right] / \mathrm{H} \alpha$ ratios, then we would expect to see the presence of these shocks reflected in increased velocity dispersions in these areas. As the emission line ratios increase in a manner consistent with shocked gas, the velocity dispersion should increase to velocities consistent with the speed of the shocks, which are of order 100-200 $\mathrm{km} \mathrm{s}^{-1}$. Lower emission line ratios consistent with gas in $\mathrm{H}$ II regions should exhibit lower velocity dispersions, typically of a few tens of $\mathrm{km} \mathrm{s}^{-1}$ (Epinat et al. 2010). In a similar study of LIRGs with IFUs, Monreal-Ibero et al. (2006, 2010) observed the correlation between excitation and velocity dispersion consistent with widespread shock excitation in a larger sample of nearby U/LIRGs and associated this excitation with tidally induced shocks.

We are able to more accurately separate the lower velocity dispersion (low- $\sigma$ ) emission consistent with $\mathrm{H}$ II regions and the higher velocity dispersion (high- $\sigma$ ) with our multiple component emission line fitting, as large portions of the gas in both systems exhibit a composite of activity both in emission line ratios and in velocity dispersion. For instance, in IC 1623 the gas falling on to the dust lane is fit well by a single fairly broad component, while gas elsewhere in the galaxy is mostly fit by either two narrow components or a narrow and broad component consistent with the complex velocity field and multiple-phase gas in this merger. 


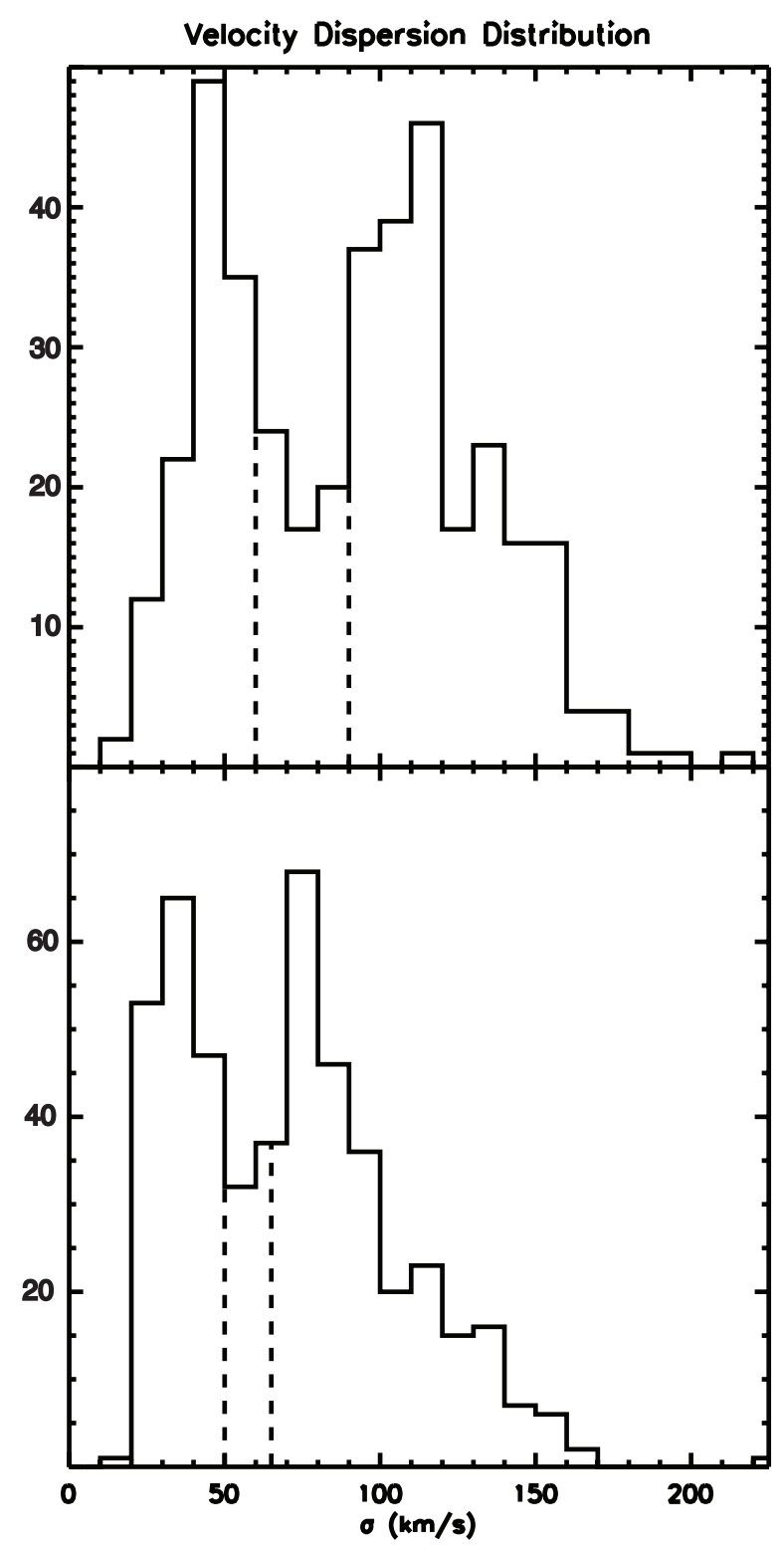

Figure 6. Distribution of emission line velocity dispersions from every individual Gaussian component fit. The bimodal distribution of velocity dispersions between the low-velocity (unresolved H II region) and high-velocity (shockexcited) components can be seen quite clearly and the cutoffs between low- and high-velocity dispersion are marked with dotted lines. The bimodal distribution in NGC 3256 is less sharply defined due to the combination of outflow and inflow.

In Figure 6, we plot a histogram of velocity dispersions for each system for every component fit by our routine. The spectrum from every spaxel was fit with either one or two Gaussian profiles with unique widths, and all of those components are plotted here. We only plot values from spaxels with emission lines of $\mathrm{S} / \mathrm{N}>5$ in all of our diagnostic lines, $\mathrm{H} \beta$, [O III], [O I], [N II], [S II], and $\mathrm{H} \alpha$. We have removed the effect of instrumental broadening from our velocity dispersions.

In both systems there is a clear peak at low- $\sigma\left(\sim 40 \mathrm{~km} \mathrm{~s}^{-1}\right.$ consistent with a large contribution from $\mathrm{H}$ II region emission due to the substantial amount of ongoing star formation). In the case of IC 1623 there is a clear bimodality between the low- $\sigma$ (H II region) and high- $\sigma$ (shocked) components. The bimodality in NGC 3256 is still present but appears less sharply delineated owing to a combination of shock excitation by the galactic wind and by bulk gas motions due to the ongoing merger.

We use the histograms in Figure 6 to establish a cutoff between the low- $\sigma$ and the high- $\sigma$ gas. For each system dashed lines show the cuts in Figure 6 which separate the emission line components into low- $\sigma$, transition, and high- $\sigma$ to better trace the different power sources. For IC 1623, we consider all gas with $\sigma<60 \mathrm{~km} \mathrm{~s}^{-1}$ as low- $\sigma$ and all gas with $\sigma>90 \mathrm{~km}$ $\mathrm{s}^{-1}$ as high- $\sigma$, with the transition falling in between these two values. Similarly, we establish cutoffs of low- $\sigma$ at $<50 \mathrm{~km} \mathrm{~s}^{-1}$ and high- $\sigma$ at $>65 \mathrm{~km} \mathrm{~s}^{-1}$ for NGC 3256 .

Figure 7 shows velocity dispersion versus emission line ratio for each individual component. Correlation between these two measures of shock excitation was explored using singlecomponent fits to IFU data of several LIRGs by Monreal-Ibero et al. (2010). The increasing velocity dispersion correlated with increasing emission line ratio corresponds to the dominance of shock excitation at high- $\sigma$, while the narrow sequence of low- $\sigma$ points (bottoming out at the instrumental resolution) corresponds to emission from $\mathrm{H}$ II region gas.

\section{ANALYSIS}

In Figure 8 we color-code the points in our diagnostic diagrams from Figure 4 using the low- $\sigma$ and high- $\sigma$ cutoffs for each system in order to separate the H II region gas from the shocked gas. The correlation of velocity dispersion with line ratio strength is still clearly seen, most obviously in the $\mathrm{N}$ II diagnostic owing to the higher $\mathrm{S} / \mathrm{N}$ of that line. More importantly, there is a clear sequence from $\mathrm{H}$ II region to LINER in the $[\mathrm{S} \mathrm{II}]$ and $\mathrm{O}_{\mathrm{I}}$ diagrams with a corresponding change from low $\sigma$ to high- $\sigma$ gas tracing the influence of shocks. The locus of the $\mathrm{H}_{\text {II }}$ region emission line gas points in the $\mathrm{N}$ II diagnostic diagram begins in the middle/low metallicity region for IC 1623 and in the higher metallicity region for NGC 3256, corresponding to the abundances of the HII regions in those galaxies.

Color coding the points accentuates the mixing sequence from pure $\mathrm{H}$ II region emission to pure shock excitation already seen in the emission line diagnostic diagrams. By combining emission line diagnostic ratios and velocity dispersion analysis of individual emission line components, we can perform a fairly conservative separation of the $\mathrm{H}$ II region gas and the shocked gas of each system and analyze them separately.

In Figure 9 we plot the line-of-sight velocity of the low- $\sigma$ and the high- $\sigma$ separately for both systems. The first thing of note is the presence of a high- $\sigma$ component over nearly the entirety of both systems clearly shocks are widespread in these galaxies. We have excluded some points from the central regions of each system where our fitting routine was unable to satisfactorily fit two Gaussians to the complex line profiles or where both emission line components are broad enough to exceed the shock cutoff. In both systems the low- $\sigma$ gas traces the HII regions which follow the rotational motion of the parent galaxies.

In IC 1623 the low- $\sigma$ gas is primarily dominated by the rotation of the eastern galaxy and is comparable to the overall shape of the rotation observed in the $\mathrm{CO}$ gas observed by Yun et al. (1994). There is a gap in low- $\sigma$ gas consistent with the dust lane seen in the HST images, as the shock excitation is correlated with the dust lane. In this region our emission line analysis only traces the tidally induced shock excited gas between the two systems because the underlying star formation of the western galaxy is completely extinguished by dust. The kinematics of the shocked gas are quite distinct, exhibiting very little correlation 


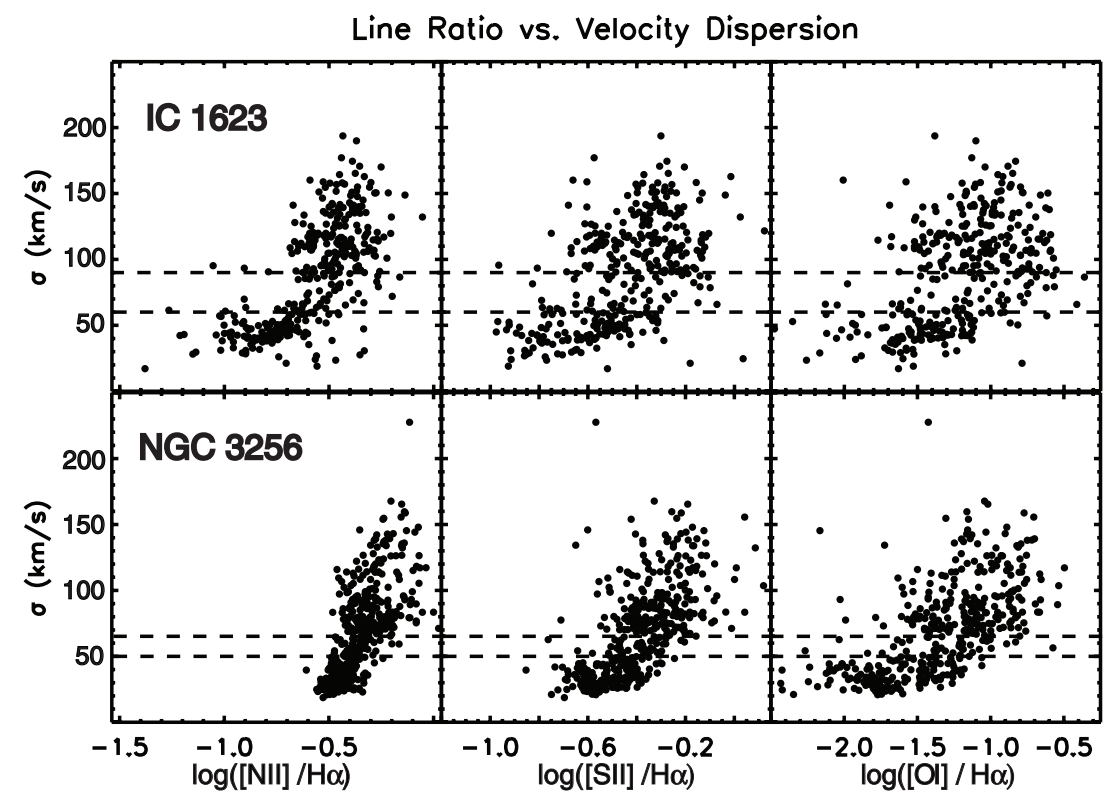

Figure 7. Plot of line ratio vs. velocity dispersion for each individual emission line component. Regions of emission line gas dominated by shock excitation show both higher emission line ratios and higher velocity dispersion resulting in the correlation seen above. A sequence of narrow component, low-excitation points corresponds to $\mathrm{H}$ II region gas of varying ionization parameters and metallicities. There is less scatter in the $[\mathrm{N} \mathrm{II}] / \mathrm{H} \alpha$ line ratio owing to the higher signal-to-noise ratio of that line, though the broader spread of $[\mathrm{S} \mathrm{II}] / \mathrm{H} \alpha$ and especially $[\mathrm{O} \mathrm{I}] / \mathrm{H} \alpha$ makes these ratios a better tracer of shock excitation.

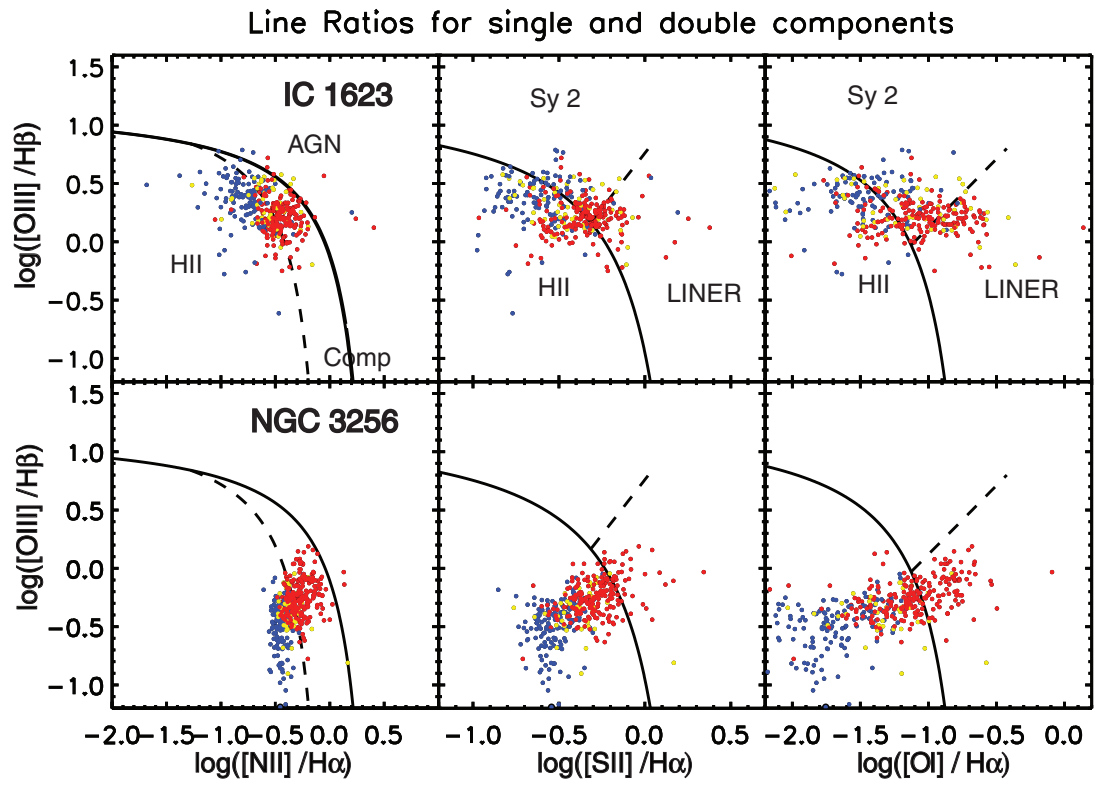

Figure 8. Same plot as Figure 4, but with points color-coded based on velocity dispersion cutoffs established in Section 5. Low- $\sigma$ points are plotted in blue, transition points in yellow, and high- $\sigma$ points in red. Clearly, the low-velocity (H II region) and the high-velocity (shocked) components are characterized by different ranges of line ratios.

(A color version of this figure is available in the online journal.)

with the rotation seen in the low- $\sigma$ gas. The shocked gas in the northern portion of IC 1623a is blueshifted up to 200 .

The kinematic distinction between the $\mathrm{H}$ II region gas and the shocked gas is more subtle in NGC 3256. Where both a shocked component and an $\mathrm{H}$ II region component are measured, however, the shocked gas is mostly blueshifted. Over the larger part of the visibly star-forming regions of NGC 3256 there is a mild shocked component blueshifted a few tens of $\mathrm{km} \mathrm{s}^{-1}$. The dust lane extinguishes the HII region gas as in IC 1623 and in spaxels where both a low- $\sigma$ and a high- $\sigma$ component are measured along the dust lane the shocked gas is generally redshifted a few tens of $\mathrm{km} \mathrm{s}^{-1}$. In addition, NGC 3256 hosts a galactic wind seen quite clearly in blueshifted $\mathrm{Na} D$ (Heckman et al. 1990, 2000). The spectra affected are within a few arcseconds of the nucleus and have a very broad blueshifted tail extending several hundred $\mathrm{km} \mathrm{s}^{-1}$ and a broader narrow component such that both components have strong line ratios and are considered high- $\sigma$ and thus excluded from the velocity plots. Finally, there is a region $\sim 8^{\prime \prime}$ in diameter of high- $\sigma$ component strongly blueshifted up to nearly $200 \mathrm{~km} \mathrm{~s}^{-1}$-lying northeast of the nucleus in a region with little dust and several closely packed star clusters, possibly a second localized outflow. 

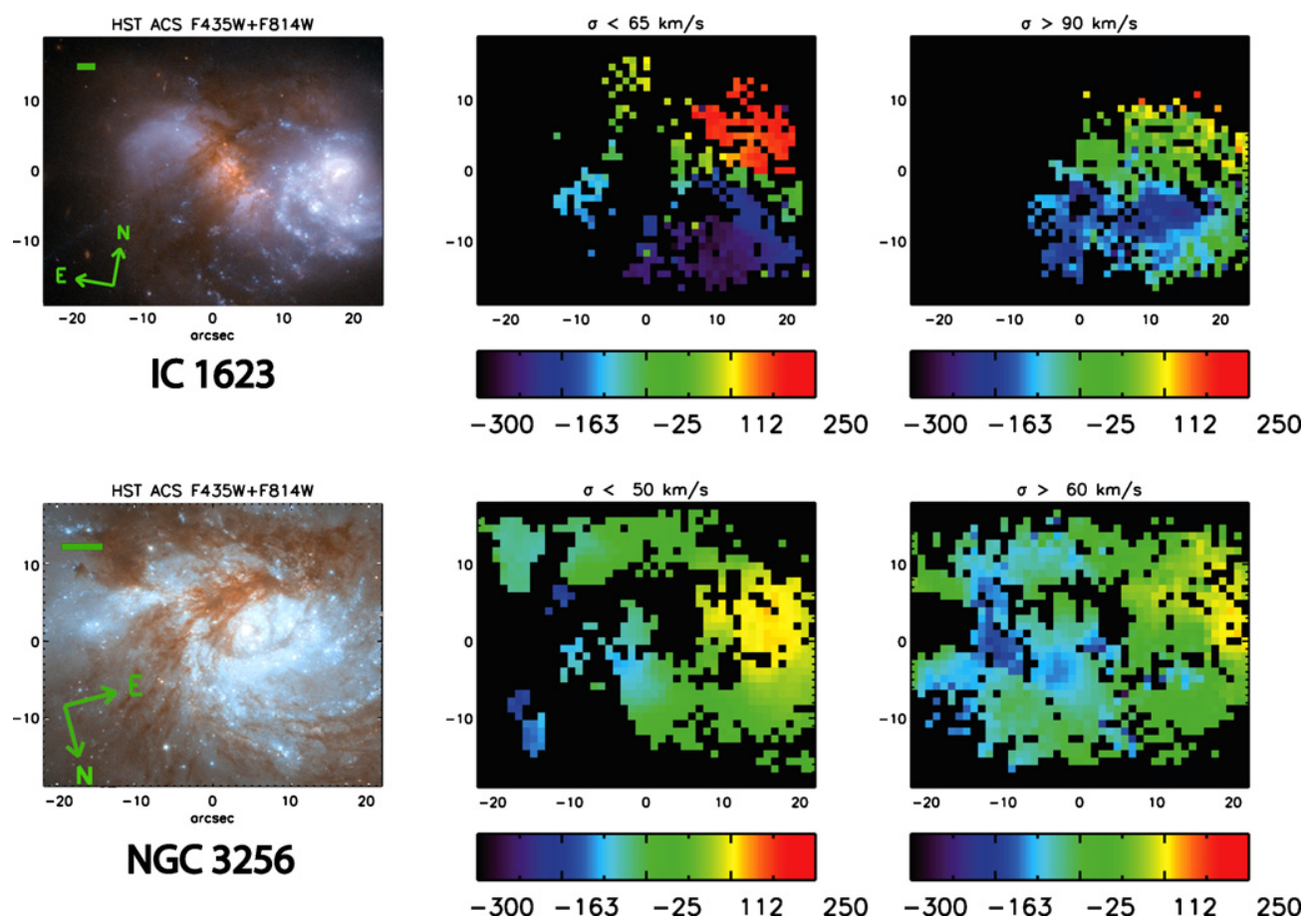

Figure 9. Line-of-sight velocities in $\mathrm{km} \mathrm{s}^{-1}$ of the $\mathrm{H}$ II region (low- $\sigma$ ) gas and the shocked (high- $\sigma$ ) gas for IC 1623 (top) and NGC 3256 (bottom). The leftmost panel is the HST ACS image from Figure 1 for reference. The H II region gas in both systems traces the rotational motion of the galaxy. The shocked gas in IC 1623 shows a dramatically different kinematic profile associated with the bulk motions of the shocked gas with components both blue- and redshifted from the H II region component. Overall the broad component in NGC 3256 is slightly blueshifted with respect to the H II region gas in the star-forming regions and slightly redshifted along the dust lanes.

(A color version of this figure is available in the online journal.)

\section{DISCUSSION}

Our analysis clearly separates the $\mathrm{H}$ II region gas from the shocked gas where both are detected. In both systems along the dust lanes and in the periphery of the galaxies where star formation either decreases or is extinguished the emission line spectra are dominated by a single, shocked component. However, our results indicate that even in the regions where emission is dominated by star formation there is still an underlying broad component associated with shocks, induced either by outflows generated by the circumnuclear star formation or by the tidal motions of the gas.

\subsection{Shock and H II Region Models}

To characterize this shock excitation we employ new slow shock models which reproduce our measured emission line ratios. These models are introduced in Farage et al. (2010) and Rich et al. (2010) and will be discussed in detail in an upcoming paper (L. J. Kewley et al. 2011, in preparation). In short we generate slow shock models with velocities consistent with our observed line widths using an updated version of the Mappings III code, originally introduced in Sutherland \& Dopita (1993). In addition, we compare $\mathrm{H}$ II region models generated using Starburst99 (Leitherer et al. 1999) and Mappings III. Both sets of models have consistent abundances of solar and twice solar for IC 1623 and NGC 3256, respectively, using the abundance set of Grevesse et al. (2010). H II models are plotted with varying ionization parameter $Q(H)$, the flux of ionizing photons divided by the hydrogen atomic density (the dimensionless ionization parameter $U=Q / c$ ), while shock models are shown at varying shock velocities.
These models are plotted in Figure 10 with the measured emission line ratios underplotted as small points. As in Figure 8, the points lying furthest to the left are more consistent with pure star formation. Our H II region models reproduce these line ratios. The data for IC1623 are consistent with a range in ionization parameters between $7.0<\log [Q(H)]<8.0$, while the higher-abundance NGC 3256 is characterized by a lower mean ionization parameter $6.75<\log [Q(H)]<$ 7.25 consistent with the dynamical models of $\mathrm{H}_{\mathrm{II}}$ regions in different metallicity environments presented by Dopita et al. (2006a, 2006b). As shocks become a more dominant ionizing mechanism and/or as the light from $\mathrm{H}$ II regions is extinguished by dust allowing shocks to dominate the line ratios migrate along the theoretical mixing sequence shown in Figure 10.

The shape of the mixing sequence evident in Figures 8 and 10 within the diagnostic diagrams is caused by the different underlying abundances in the two systems. In IC 1623 the lowerabundance gas means as shock excitation begins to dominate the line ratios move horizontally across the diagrams to the LINER region. The relatively higher abundance of NGC 3256 however creates a tilted sequence with the pure $\mathrm{H}$ II region points beginning in a different region of the diagnostic diagrams. This difference is most noticeable in the $\mathrm{N}_{\text {II }}$ diagnostic diagram where the different metallicities are most widely separated in line ratio space.

Varying the fractional contribution of shock excitation within the parameters of our models recovers the observed emission line ratios quite well. It is important to note in Figure 10 that the line ratios are sensitive to as little as $20 \%$ shock excitation. The observed ratios in IC 1623 cover nearly the entire mixing sequence, implying that the various regions sampled in the galaxy cover a range of shock velocities, though most of the 


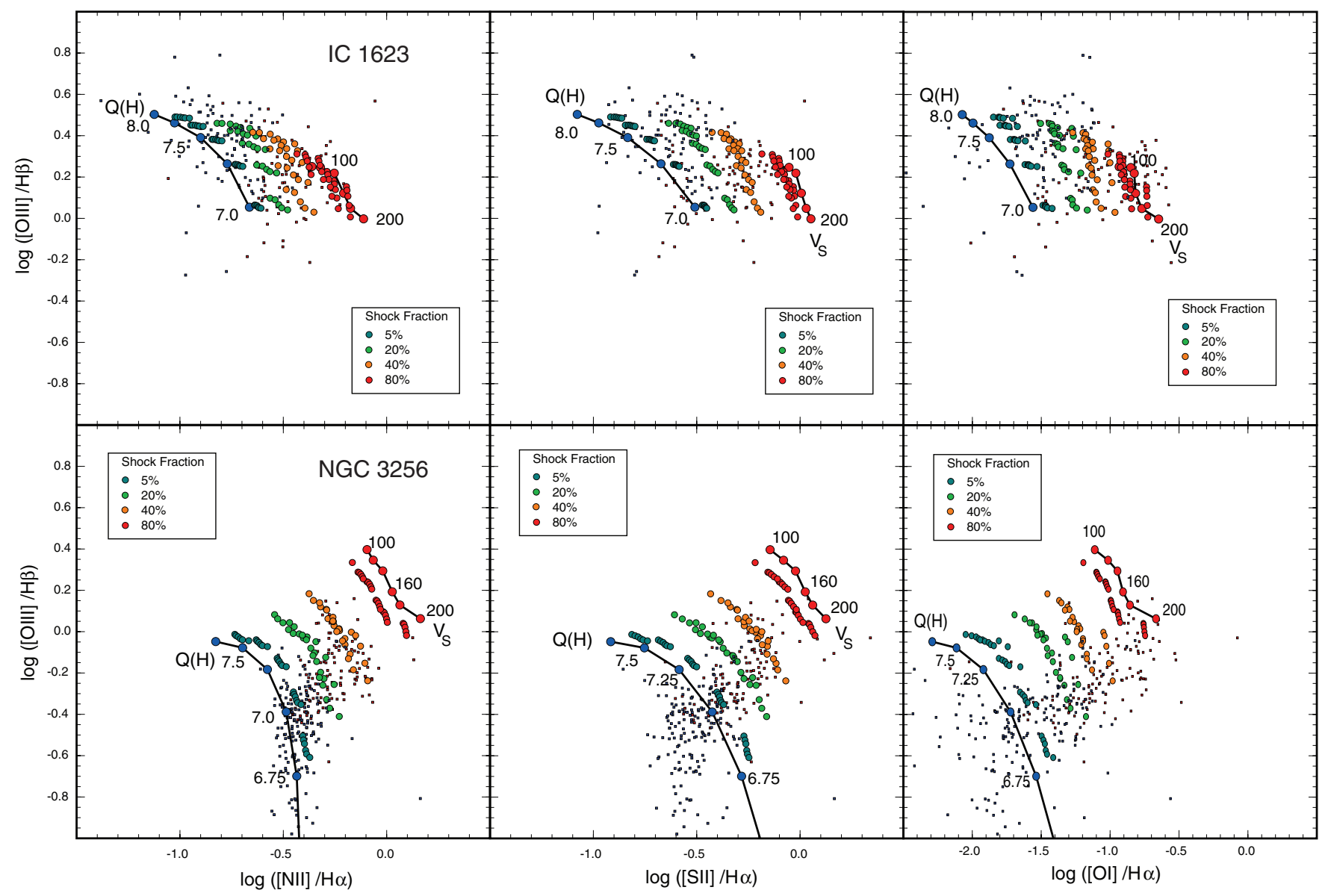

Figure 10. Replotted measured emission line ratios (small points) with our $\mathrm{H}$ II region and shock models overlaid. The $\mathrm{H}$ II region models are larger blue circles connected by a black line and are plotted with varying ionization parameter $(Q)$ and adopting abundances of solar and twice solar, respectively, for IC 1623 and NGC 3256. The fully preionized slow shock models are plotted as larger red circles and are also connected by a black line along a sequence of increasing shock velocity. The colored points in between show a mixing sequence from pure $\mathrm{H}$ II region to pure shock excitation.

(A color version of this figure is available in the online journal.)

points near the end of the mixing sequence correspond to $100-140 \mathrm{~km} \mathrm{~s}^{-1}$. In NGC 3256 the line ratios both within the mixing sequence and near the pure shock models are consistent with shock velocities of $\sim 160-200 \mathrm{~km} \mathrm{~s}^{-1}$. In both systems the terminus of our fastest shock models shows remarkable agreement with the strongest measured emission line ratios.

\subsection{Global Energetic Considerations}

It is worthwhile to make a few simple calculations to quantify the impact that shocks have on dissipating the energy of the infalling gas in merging systems. Here we focus on the wellstudied NGC 3256, where previous wide-scale observations facilitate these calculations.

Once the shocked emission in our data has been isolated we can use the measured $\mathrm{H} \alpha$ flux provided solely by shocks to extrapolate the total shock luminosity. For this calculation we make the simple assumption that the total $F_{\mathrm{H}_{\alpha}}$ measured in any line components above the shock velocity dispersion threshold is caused by shocks. We use the plot provided in Rich et al. (2010) to approximate the total shock luminosity from $L_{\mathrm{H}_{\alpha}}$ originating in shocks. This provides an upper limit to the total shock luminosity of $80 \times L_{\mathrm{H}_{\alpha}}$. For NGC 3256 this corresponds to an approximate global shock luminosity of $L_{\text {shock }} \sim 8 \times 10^{42} \mathrm{erg} \mathrm{s}^{-1}$.
Working with this luminosity as a starting point we next consider the total gas available as well as the mass and size of the system. English \& Freeman (2003) and English et al. (2003) report an $I$-band (stellar) mass within $70^{\prime \prime}$ of the system of $(2.5 \pm 0.5) \times 10^{10} h^{-1} M_{\odot}$ and a total $\mathrm{HI}$ gas mass of $3.5 \times 10^{9} h^{-2} M_{\odot}, 75 \%$ of which resides in tails extending to a total distance of $42 h^{-1} \mathrm{kpc}$. This does not take into account the molecular gas mass, which may be larger. Adopting our value of $h=0.7$ provides a dynamical timescale of $\sim 800 \mathrm{Myr}$ and a total binding energy for the $\mathrm{H}$ I gas of $\sim 8 \times 10^{56} \mathrm{erg}$.

The SFR can be approximated using $\mathrm{L}_{\mathrm{H}_{\alpha}}$ and $\mathrm{L}_{\mathrm{IR}}$ (Kennicutt 1998; Dopita et al. 2005, 2003; Calzetti et al. 2007; Kennicutt et al. 2009). Lira et al. (2002) estimate the total $\mathrm{F}_{\mathrm{H}_{\alpha}} \sim 2 \times$ $10^{-11} \mathrm{erg} \mathrm{s}^{-1} \mathrm{~cm}^{-2}$, with data from Lípari et al. (2000) using a larger aperture than is available to our IFS data. Applying a total reddening correction of $E(B-V)=0.5$, approximated using maps of the Balmer decrement from our data cubes, yields $\mathrm{L}_{\mathrm{H}_{\alpha}} \sim 1.2 \times 10^{43} \mathrm{erg} \mathrm{s}^{-1}$. Assuming a fractional contribution to $\mathrm{H} \alpha$ from shocks of $\sim 30 \%$, consistent with our IFS aperture, the total SFRs are $\sim 66 M_{\odot} \mathrm{yr}^{-1}$ and $\sim 75 M_{\odot} \mathrm{yr}^{-1}$ using $\mathrm{L}_{\mathrm{H}_{\alpha}}$ and $\mathrm{L}_{\mathrm{IR}}$, respectively. The addition of both extinction correction and an adjustment due to shock contribution yields a better agreement in the SFRs. If an SFR of $75 M_{\odot} \mathrm{yr}^{-1}$ persists the available $\mathrm{H}$ I gas can fuel the starburst at this rate for $\sim 100 \mathrm{Myr}$.

For shocks to aid in removing kinetic energy from the infalling gas, we consider the amount of time it would take for the shock 


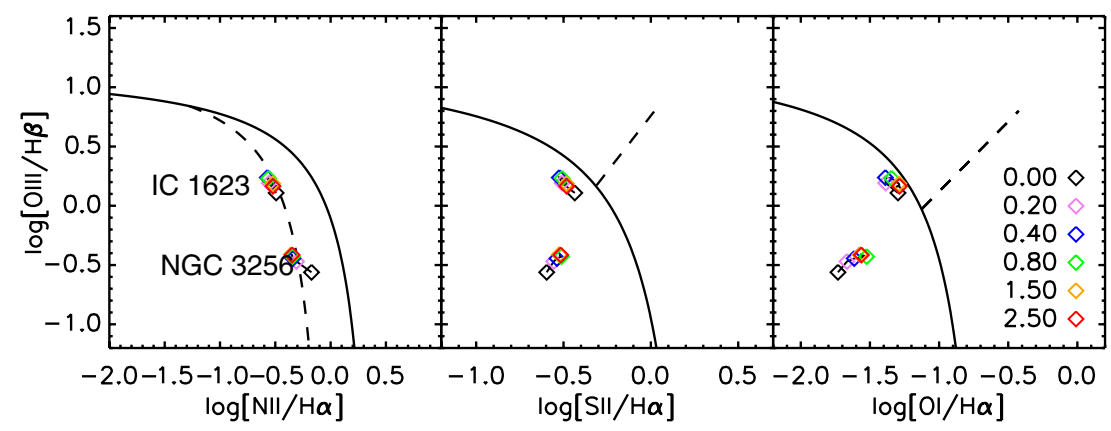

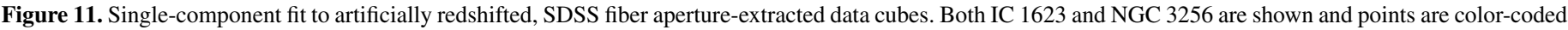
according to redshift. The points do not shift more than $\sim 0.4$ dex. NGC 3256 moves from a composite classification to an H II classification in the N II diagnostic. (A color version of this figure is available in the online journal.)

luminosity to dissipate about twice the binding energy of the available $\mathrm{H}_{\mathrm{I}}$ gas, or $\sim 10^{56} \mathrm{erg}$. If the entirety of the shock luminosity of $L_{\text {shock }} \sim 8 \times 10^{42} \mathrm{erg} \mathrm{s}^{-1}$ acts in this way, the kinetic energy dissipation timescale is $\sim 6 \mathrm{Myr}$, significantly less than the dynamical timescale, implying that shocks may act as a very efficient mechanism for removing kinetic energy from the infalling gas, allowing it to feed the central starburst. English \& Freeman (2003), however, do not consider inclination effects in their mass calculations, which would effectively increase this timescale.

\subsection{Star Formation Rate}

The $\mathrm{H} \alpha$ flux is often used as a direct proxy for SFR in galaxies, typically assuming the relation of Kennicutt (1998). If one assumes that all of the high- $\sigma$ shocked emission in each system is coming from pure shock excitation, a simple total fractional contribution from shocks and thus the effect on the measure of SFR can be estimated.

Internal extinction will also impact the measurement of $\mathrm{H} \alpha$-derived SFR and may affect regions of shock excitation differently than regions dominated by $\mathrm{H}$ II photoionization. When comparing the Balmer decrements from our data for both IC 1623 and NGC 3256, the average difference between these regions is negligible. In some portions of the galaxy where both high- and low- $\sigma$ components are seen, the extinction in the starburst component is slightly stronger than in the shocked component, though the average difference in $E(B-V)$ is comparable to the intrinsic spread in values.

After applying the velocity dispersion cutoffs, the total $\mathrm{H} \alpha$ flux of the high- $\sigma$ emission line gas accounts for as much as $1 / 3$ of the total $\mathrm{H} \alpha$ emission, which would decrease an estimated SFR by $\sim 30 \%$. Clearly shocked emission must be considered when SFR measurements are made from $\mathrm{H} \alpha$ in galaxies with starburst-driven winds and merger widespread shock excitation.

\subsection{Aperture and Redshift}

As shocks contribute fractionally to the emission line fluxes over the majority of the galaxy and dominate the total flux in some off-nuclear regions, care must be taken when deriving quantities from the emission line spectra. In order to better understand the effect of aperture size and its changing physical scale with redshift, we artificially redshifted our data cubes while extracting a pseudo-fiber spectrum from the peak in median rest-frame red light flux.

We choose SDSS as our point of comparison, using a $3^{\prime \prime}$ fiber size for our aperture extractions and rebinning our spectra in wavelength to match the approximate $R \sim 1800$ spectroscopic resolution of SDSS. We maintained a spaxel size of $1^{\prime \prime}$ while spatially rebinning and dimming the data cubes to correspond to $z$ of $0.2,0.4,0.8,1.5$, and 2.5. After extracting a composite pseudo-fiber spectrum at each redshift, we apply our fitting routine using both one- and two-component Gaussian fits to emission lines. The measured emission line ratios for each redshift and each component are shown in Figure 11. Asymmetries in the emission lines are in some cases fit somewhat better by two components, but the overall separation between the two components is $\sim 100 \mathrm{~km} \mathrm{~s}^{-1}$, within the simulated resolution.

As we redshift our data, the emission line ratios do not change by more than 0.4 dex at the most. Most of this shift occurs after the first redshift owing to the binning of a large part of the galaxies emission. Both galaxies move from a more composite to a more starburst-like classification in the $\mathrm{N}$ II diagram. The two-component fit is more difficult to interpret, but generally the broader component in both systems moves to the right in the diagnostic diagrams (more shock-like), while the narrower component moves to the left (more $\mathrm{H}$ II region-like). Because of the low separation in velocity space, the interpretation of the secondary component is probably unreliable.

With such an SDSS fiber aperture at the highest simulated redshifts $(z=1.5,2.5)$ the spectra become a sum of nearly all of the emission line gas in the system and the total emission line fluxes of the weaker lines decrease to a few $\times 10^{-17} \mathrm{erg} \mathrm{s}^{-1} \mathrm{~cm}^{-2}$ or less, making them marginally detectable. Proportionally more of the high- $\sigma$ gas is of lower surface brightness, so that while more of the outer regions dominated by high- $\sigma$ shocked gas are co-added, their total surface brightness drops, countering this. The total velocity dispersions of the narrow portion of the emission lines also become broader as more $\mathrm{H} \mathrm{II}$ region ensembles are co-added, increasing to higher- $\sigma$ of $\sim 100$ consistent with those measured in high- $z$ galaxies. This effect was noted using IFU observations by Epinat et al. (2010) and causes difficulty in discerning between large dispersion clumps and effects and the co-adding of several low- $\sigma$ regions at high redshift. This is especially noticeable in IC 1623 which has a complex velocity structure across the majority of the brightest portions of the galaxy.

Because of these effects, separating the shocked gas component from the star-forming component within global spectra is difficult at the highest $z$. Improvement can be achieved with higher spatial and spectral sampling coupled with deep observations, though the purely shocked high- $\sigma$ regions associated with the dust lanes in NGC 3256 and IC 1623 are of lower surface brightness making them difficult to detect even if the global spectra show composite behavior. 


\section{CONCLUSION}

Using IFU emission line data of two nearby LIRGs, we have found a bimodal distribution of velocity dispersions and emission line ratios indicative of composite activity. This composite emission can be explained by a combination of shocks and star formation. In order to separate the shocked component from the purely star-forming component we establish cutoffs in velocity dispersion to distinguish the shocked higher- $\sigma$ component from the $\mathrm{H}$ II region-like lower- $\sigma$ gas. Once this distinction has been made, the $\mathrm{H}$ II region-like gas can be used to calculate derived quantities based on $\mathrm{H}$ II region emission line data such as SFR and metallicity. The properties and overall contribution of the shocked gas can also be inferred from the emission line data as well. We have provided such an example using low-velocity shock models which accurately reproduce the observed emission line ratios at the extreme end of the emission line ratio diagnostics. The shape of the mixing sequences between these models is directly influenced by the gas abundance and our H II region models imply a distribution of $Q(H)$ consistent with the dynamical models for H II regions by Dopita et al. (2006a, 2006b).

We urge caution when interpreting emission line data of both nearby and high- $z$ galaxies, especially when spatial information is limited. Traditionally, the classification of a galaxy relies on a nuclear spectrum or a globally extracted aperture in the case of more distant systems. Using this method our systems would be classified as composite locally and starburst at higher- $z$, though this classification is affected by the size and position of the aperture extracted and the morphology of the system. Slit spectra provide a slightly more complete picture though the alignment and size of the slit and the size and distance of the system may complicate interpretation. This is especially true at high redshift $(z \sim 1.5-3)$ where a slight misalignment of the slit coupled with the drastic reduction in surface brightness of most features makes such measurements difficult and may miss the complete picture.

Our data clearly illustrate the importance of shocks in merging galaxies - not only do they affect quantities derived from spectroscopy, they also act as a useful mechanism for dissipating the kinetic energy and angular momentum of the infalling gas in merging systems. As a dissipative mechanism, radiative shocks aid in driving the gas to the center of the galaxy in order to fuel the ongoing central starburst and keeping it in an increasingly deep potential well, and presumably feed the eventual AGN. Presumably, a portion of the shock excitation is generated by galactic winds as the merger progresses, though even a few percent of the total shock luminosity we calculate is sufficient to dissipate the binding energy on a timescale shorter than the dynamical timescale (10-100 Myr versus $800 \mathrm{Myr}$ ). As the gas is continually driven inward and shocked by tidal forces, galactic outflows coupled with star formation will also compete to use up the gas on the scale of a few hundred million years or so.

Our work also illustrates the utility of IFS in accurately decomposing the complex aspects of a merger. Not only is the full spatial picture of many properties available with an IFU observation, it is easier to separate the widespread shocked component and to trace its spatial and kinematic structure. Once this information has been isolated, the derived quantities of the $\mathrm{H}$ II region emission can be more accurately calculated and the detailed properties of the shocked gas itself can be investigated. This is especially important when calculating metallicities from strong-line calibrations, a quantity of particular interest in merging galaxies locally and in the early universe. A forthcoming paper (L. J. Kewley et al. 2011, in preparation) will provide a theoretical framework for the effect of shock emission on such measurements and discuss the mixing sequence in galaxies such as those presented in this paper. We also plan to apply this careful separation of shocked gas from $\mathrm{H}$ II region emission where necessary to the measurement of metallicities in a larger sample of IFU data of merging galaxies in a separate paper (J. A. Rich et al. 2011, in preparation).

We thank the referee for his/her helpful comments, which helped clarify several points in this paper. M.A.D. acknowledges the support from the Australian Department of Science and Education (DEST) Systemic Infrastructure Initiative grant and from an Australian Research Council (ARC) Large Equipment Infrastructure Fund (LIEF) grant LE0775546 which together made possible the construction of the WiFeS instrument. M.A.D. also thanks the Australian Research Council (ARC) for support under Discovery project DP0664434. M.A.D., L.J.K., and J.A.R. acknowledge ARC support under Discovery project DP0984657. This research has made use of the NASA/IPAC Extragalactic Database (NED) which is operated by the Jet Propulsion Laboratory, California Institute of Technology, under contract with the National Aeronautics and Space Administration. This research has also made use of NASA's Astrophysics Data System, and of SAOImage DS9 (Joye \& Mandel 2003), developed by the Smithsonian Astrophysical Observatory.

\section{REFERENCES}

Alonso-Herrero, A., Rieke, G. H., Rieke, M. J., Colina, L., Pérez-González, P. G., \& Ryder, S. D. 2006, ApJ, 650, 835

Armus, L., Heckman, T. M., \& Miley, G. K. 1989, ApJ, 347, 727

Armus, L., et al. 2009, PASP, 121, 559

Arribas, S., Bushouse, H., Lucas, R. A., Colina, L., \& Borne, K. D. 2004, AJ, 127,2522

Baldwin, J. A., Phillips, M. M., \& Terlevich, R. 1981, PASP, 93, 5

Barnes, J. E., \& Hernquist, L. 1992, ARA\&A, 30, 705

Barnes, J. E., \& Hernquist, L. 1996, ApJ, 471, 115

Bessell, M. S. 1999, PASP, 111, 1426

Boeker, T., Storey, J. W. V., Krabbe, A., \& Lehmann, T. 1997, PASP, 109, 827

Calzetti, D., Harris, J., Gallagher, J. S., III, Smith, D. A., Conselice, C. J., Homeier, N., \& Kewley, L. 2004, AJ, 127, 1405

Calzetti, D., et al. 2007, ApJ, 666, 870

Colina, L., Arribas, S., \& Monreal-Ibero, A. 2005, ApJ, 621, 725

de Vaucouleurs, G., \& de Vaucouleurs, A. 1961, Mem. R. Astron. Soc., 68, 69

Dopita, M., Hart, J., McGregor, P., Oates, P., Bloxham, G., \& Jones, D. 2007, Ap\&SS, 310, 255

Dopita, M., et al. 2010, Ap\&SS, 327, 325

Dopita, M. A., Groves, B. A., Sutherland, R. S., \& Kewley, L. J. 2003, ApJ, 583,727

Dopita, M. A., Kewley, L. J., Heisler, C. A., \& Sutherland, R. S. 2000, ApJ, 542,224

Dopita, M. A., et al. 2005, ApJ, 619, 755

Dopita, M. A., et al. 2006a, ApJ, 647, 244

Dopita, M. A., et al. 2006b, ApJS, 167, 177

Doyon, R., Nadeau, D., Joseph, R. D., Goldader, J. D., Sanders, D. B., \& Rowlands, N. 1995, ApJ, 450, 111

Elbaz, D., Cesarsky, C. J., Chanial, P., Aussel, H., Franceschini, A., Fadda, D., \& Chary, R. R. 2002, A\&A, 384, 848

English, J., \& Freeman, K. C. 2003, AJ, 125, 1124

English, J., Norris, R. P., Freeman, K. C., \& Booth, R. S. 2003, AJ, 125, 1134

Epinat, B., Amram, P., Balkowski, C., \& Marcelin, M. 2010, MNRAS, 401, 2113

Farage, C. L., McGregor, P. J., Dopita, M. A., \& Bicknell, G. V. 2010, ApJ, 724, 267

Genzel, R., et al. 1998, ApJ, 498, 579

González Delgado, R. M., Cerviño, M., Martins, L. P., Leitherer, C., \& Hauschildt, P. H. 2005, MNRAS, 357, 945

Grevesse, N., Asplund, M., Sauval, A. J., \& Scott, P. 2010, Ap\&SS, 328, 179

Heckman, T. M., Armus, L., \& Miley, G. K. 1990, ApJS, 74, 833 
Heckman, T. M., Lehnert, M. D., Strickland, D. K., \& Armus, L. 2000, ApJS, 129,493

Hinshaw, G., et al. 2009, ApJS, 180, 225

Jabran Zahid, H., Kewley, L. J., \& Bresolin, F. 2011, ApJ, 730, 137

Joye, W. A., \& Mandel, E. 2003, in ASP Conf. Ser. 295, Astronomical Data Analysis Software and Systems XII, ed. H. E. Payne, R. I. Jedrzejewski, \& R. N. Hook (San Francisco, CA: ASP), 489

Kauffmann, G., et al. 2003, MNRAS, 346, 1055

Kennicutt, R. C., Jr. 1998, ApJ, 498, 541

Kennicutt, R. C., et al. 2009, ApJ, 703, 1672

Kewley, L. J., Groves, B., Kauffmann, G., \& Heckman, T. 2006, MNRAS, 372 , 961

Kewley, L. J., Heisler, C. A., Dopita, M. A., \& Lumsden, S. 2001, ApJS, 132, 37

Kewley, L. J., Rupke, D., Jabran Zahid, H., Geller, M. J., \& Barton, E. J. 2010, ApJ, 721, L48

Knop, R. A., Soifer, B. T., Graham, J. R., Matthews, K., Sanders, D. B., \& Scoville, N. Z. 1994, AJ, 107, 920

Le Floc'h, E., et al. 2005, ApJ, 632, 169

Lehnert, M. D., \& Heckman, T. M. 1996, ApJ, 462, 651

Leitherer, C., et al. 1999, ApJS, 123, 3

Lípari, S., Díaz, R., Taniguchi, Y., Terlevich, R., Dottori, H., \& Carranza, G. 2000, AJ, 120, 645

Lípari, S., et al. 2004a, MNRAS, 355, 641

Lípari, S. L., et al. 2004b, MNRAS, 354, L1

Lira, P., Ward, M., Zezas, A., Alonso-Herrero, A., \& Ueno, S. 2002, MNRAS, 330, 259

Markwardt, C. B. 2009, in ASP Conf. Ser. 411, Astronomical Data Analysis Software and Systems XVIII, ed. D. A. Bohlender, D. Durand, \& P. Dowler (San Francisco, CA: ASP), 251
Moiseev, A., Karachentsev, I., \& Kaisin, S. 2010, MNRAS, 403, 1849

Monreal-Ibero, A., Arribas, S., \& Colina, L. 2006, ApJ, 637, 138

Monreal-Ibero, A., Arribas, S., Colina, L., Rodríguez-Zaurín, J., AlonsoHerrero, A., \& García-Marín, M. 2010, A\&A, 517, A28

Mould, J. R., et al. 2000, ApJ, 529, 786

Moustakas, J., \& Kennicutt, R. C., Jr. 2006, ApJS, 164, 81

Neff, S. G., Ulvestad, J. S., \& Campion, S. D. 2003, ApJ, 599, 1043

Rich, J. A., Dopita, M. A., Kewley, L. J., \& Rupke, D. S. N. 2010, ApJ, 721, 505

Rupke, D. S., Veilleux, S., \& Sanders, D. B. 2005, ApJS, 160, 115

Rupke, D. S. N., Kewley, L. J., \& Barnes, J. E. 2010a, ApJ, 710, L156

Rupke, D. S. N., Kewley, L. J., \& Chien, L. 2010b, ApJ, 723, 1255

Sakamoto, K., Ho, P. T. P., \& Peck, A. B. 2006, ApJ, 644, 862

Sanders, D. B., Mazzarella, J. M., Kim, D., Surace, J. A., \& Soifer, B. T. 2003, AJ, 126, 1607

Scoville, N. Z., et al. 2000, AJ, 119, 991

Sharp, R. G., \& Bland-Hawthorn, J. 2010, ApJ, 711, 818

Shopbell, P. L., \& Bland-Hawthorn, J. 1998, ApJ, 493, 129

Sutherland, R. S., \& Dopita, M. A. 1993, ApJS, 88, 253

Toomre, A., \& Toomre, J. 1972, ApJ, 178, 623

Veilleux, S., Kim, D., Sanders, D. B., Mazzarella, J. M., \& Soifer, B. T. 1995, ApJS, 98, 171

Veilleux, S., \& Osterbrock, D. E. 1987, ApJS, 63, 295

Veilleux, S., \& Rupke, D. S. 2002, ApJ, 565, L63

Veilleux, S., Shopbell, P. L., Rupke, D. S., Bland-Hawthorn, J., \& Cecil, G. 2003, AJ, 126, 2185

White, S. D. M. 1979, MNRAS, 189, 831

Yun, M. S., Scoville, N. Z., \& Knop, R. A. 1994, ApJ, 430, L109

Yuan, T., Kewley, L. J., \& Sanders, D. B. 2010, ApJ, 709, 884

Zakamska, N. L. 2010, Nature, 465, 60 